International League Against Epilepsy (ILAE) epidemiologic guidelines [2]. Definite and probable cases of seizures and epilepsy were classified according to 2017 ILAE classification systems as focal, generalized or unknown [3, 4]. Data were analysed using SPSS, version 24.

Results From a population of 542,869 adults and children, 1942 potential cases were identified, of whom 611 were excluded as neonatal or febrile seizures, did not meet the geographic criteria or had a previous diagnosis of seizures or epilepsy. Incidence rates of first seizure (both provoked and unprovoked) was 102 per 100,000 population, of new diagnosis of epilepsy was 64 per 100,000, and of seizure mimics was 96 per 100,000. In concordance with most international studies, age-specific incidence rates for both first seizures and new diagnosis of epilepsy demonstrated a bimodal distribution, with highest rates in the very young and in later life. As expected, the most commonly encountered seizure mimic was syncope $(30 \%)$.

Conclusion We applied a rigorous study protocol for investigation of the incidence of first seizures, new diagnosis of epilepsy and seizure mimics in a geographically defined region which is adherent to recently published international guidelines for epidemiological studies and epilepsy classification. This study highlights the significant burden that seizure mimics place on diagnostic services given that they occur as frequently as first seizures.

\section{P72 CARING FOR CRITICALLY ILL WOMEN IN OBSTETRICS IN IRELAND: 2014-2016}

E Manning*, P Corcoran, I Farrell, S Leitao, R Greene. Obstetrics and Gynaecology, National Perinatal Epidemiology Centre, Cork, Ireland

\subsection{6/jech-2019-SSMabstracts.223}

Background The provision of safe maternal critical care requires resource planning and development of relevant competencies among healthcare professionals. However, there are no national data recording the activity of higher levels of care in obstetrics. The objective of this study was to establish the incidence, location of care and underlying maternal morbidity associated with Critical Care in Obstetrics in Ireland.

Methods For 2014-2016, 15 Irish maternity units provided anonymised data on pregnant or recently pregnant women requiring Level 2 Care (invasive monitoring or support for a single failing organ system) or Level 3 Care (requiring mechanical ventilation alone or support of two or more organ systems). Morbidities were classified using both the World Health Organisation and the National Perinatal Epidemiology Centre definitions of severe maternal morbidity.

Results Among 124,135 maternities, 900 women required Level 2 Care (7.3 per 1,000 maternities) and 61 women required Level 3 Care (0.5 per 1,000 maternities). While Level 3 Care was provided in an ICU facility, the location of Level 2 Care varied by maternity unit - the smaller the unit, the greater the utilisation of the ICU. Respectively, hypertensive disorders and obstetric haemorrhage affected $54.2 \%$ and $27.3 \%$ of women requiring Level 2 Care and $11.5 \%$ and $44.3 \%$ of women requiring Level 3 Care. The need for higher level of care was not predictable in approximately half of the women. All woman requiring Level 3 Care and $37.1 \%$ of women requiring Level 2 Care met the criteria of organ dysfunction as specified by the national clinical audit of severe maternal morbidity.
Conclusion A significant number of women requiring Level 2 Care do not experience organ dysfunction as their clinical needs were identified and treated before organ dysfunction occurred. Thus, there are limitations of existing classification systems on severe maternal morbidity in quantifying level of care provided. The variation in location of Level 2 Care has implications for staff training in both maternity units and ICU.

\section{P73 IDENTIFYING THE ACTIVE INGREDIENTS IN IMPLEMENTATION: QUALITATIVE CONTENT ANALYSIS OF THE OVERLAP BETWEEN BEHAVIOUR CHANGE TECHNIQUES AND IMPLEMENTATION STRATEGIES}

${ }^{1} S$ McHugh*, ${ }^{2}$ Presseau, ${ }^{3} \mathrm{C}$ Luecking, ${ }^{3} \mathrm{~B}$ Powell. ${ }^{1}$ School of Public Health, University College Cork, Cork, Ireland; ${ }^{2}$ School of Epidemiology and Public Health, University of Ottawa, Ottawa, Canada; ${ }^{3}$ Department of Health Policy and Management, University of North Carolina, Chapel Hill, USA

\subsection{6/jech-2019-SSMabstracts.224}

Background Evidence-based healthcare innovations require complementary evidence-based implementation strategies to support their translation into practice. Efforts to test, refine and replicate implementation strategies are frustrated by insufficient description. Our aim was to examine the extent to which implementation strategies could be specified using the Behaviour Change Technique (BCT) taxonomy, a behavioural science tool for describing the active ingredients of interventions.

Methods The data source was a compilation of 73 implementation strategies, developed through evidence synthesis and expert consensus. The definition of each strategy $(n=73)$ was deductively coded using the BCT Taxonomy, containing 93 discrete techniques. A typology was developed iteratively to categorise the extent of overlap between strategies and BCTs. The number of BCTs per strategy and extent of overlap was estimated. In the next stage, 3 experts will independently rate 1) their level of agreement with the categorisation and 2) level of agreement with the BCT(S) identified within each strategy.

Results During preliminary analysis, 87 BCTs were coded across 73 strategies (average 1.2 per strategy). Five types of overlap were identified. For $8 \%$ of strategies $(n=6)$, there was direct overlap between the strategy description and BCT (e.g. strategy: remind clinicians/BCT: prompts and cues). For 36\% of strategies $(n=26)$, there was at least 1 BCT clearly subsumed under the strategy description which could be used to guide initial operationalisation (e.g. strategy: provide clinical supervision/BCT: restructure social environment). For $26 \%$ of strategies $(n=19)$, a BCT(s) was probably subsumed under the strategy given its definition and/or title but other BCTs were possible depending on how the strategy is operationalised (e.g. strategy: visit other implementation sites/BCT: social comparison). For $11 \%(n=8)$, there were no BCTs clearly indicated in the strategy definition or title (e.g. strategy: make training dynamic). Finally, $19 \%$ of strategies $(n=14)$ did not focus on behaviour change to support implementation (e.g. strategy: access new funding).

Conclusion Many implementation strategies require further specification in order to apply them in a setting, relying on assumptions and inference on the part of the intervention developer, be it researcher or practitioner. This creates an 